\title{
Immunotherapy in malignant pleural mesothelioma: a review of literature data
}

\author{
Jessica Menis ${ }^{1,2}$, Giulia Pasello ${ }^{2}, J_{0 r d i}$ Remon $^{3}$ \\ ${ }^{1}$ Department of Surgery, Oncology and Gastroenterology, University of Padova, Padova, Italy; ${ }^{2}$ Medical Oncology Department, Istituto Oncologico \\ Veneto IRCCS, Padova, Italy; ${ }^{3}$ Department of Medical Oncology, Centro Integral Oncológico Clara Campal (HM-CIOCC), Hospital HM Delfos, \\ HM Hospitales, Barcelona, Spain \\ Contributions: (I) Conception and design: All authors; (II) Administrative support: All authors; (III) Provision of study materials or patients: All \\ authors; (IV) Collection and assembly of data: All authors; (V) Data analysis and interpretation: All authors; (VI) Manuscript writing: All authors; (VII) \\ Final approval of manuscript: All authors. \\ Correspondence to: Jessica Menis. Department of Surgery, Oncology and Gastroenterology, University of Padova, Padova, Italy. \\ Email: j16menis@gmail.com.
}

\begin{abstract}
Malignant pleural mesothelioma (MPM) is a rare, aggressive cancer of the pleural surface, associated with asbestos exposure, whose incidence is still growing in some areas of the world. MPM is still considered a rare and an orphan disease with an unchanged median overall survival (OS) ranging from 8 to 14 months and no treatment advances in the last 15 years both in local and advanced disease. In the recent years, chronic inflammation of the mesothelium together with local tumor suppression plays a major role in the malignant transformation. Also, significant heterogeneity in both tumor and the microenvironment is at the basis of MPM biology. Preclinical data have demonstrated the immunogenicity and the lack of an effective antitumor response by the immune system in MPM thus paving the way to the development of immune therapeutics in this disease. Still there is no clear evidence of any predictive biomarker so that, given the close interaction between the immune infiltrate and mesothelial cells, a number of trials are ongoing to investigate the role and prognostic value of the immune microenvironment. In this review we summarize the rationale for immune therapeutics development in MPM, as well as, the relevant literature and ongoing trials of immune checkpoint inhibitors (ICIs) and vaccines used as both first-line treatment and beyond.
\end{abstract}

Keywords: Mesothelioma; immunotherapy; vaccine; immune checkpoint inhibitors (ICIs)

Submitted May 15, 2020. Accepted for publication Jun 04, 2021.

doi: $10.21037 /$ tlcr-20-673

View this article at: http://dx.doi.org/10.21037/tlcr-20-673

\section{Introduction}

Malignant pleural mesothelioma (MPM) is included in the category of rare cancers, with an annual incidence in the European Union of 2.14 cases per 100,000 persons per year (1). The prognosis of MPM has not improved in the last 15 years, with a five-year overall survival (OS) of $5 \%$ (2), being thus considered an orphan disease. MPM is clearly related to asbestos exposure and although asbestos banner in Western countries occurred in the 80's decade, the incidence of MPM is still raising since the time elapsed between exposure and disease onset is typically more than
30 years. Standard systemic treatment is still pemetrexed plus platinum-based chemotherapy (3), and in very limited and highly selected patients surgery and radiation may take place in the therapeutic trimodal treatment strategy (3-5). The role of maintenance is controversial: according to recent results from phase II ALIANCE trial, maintenance treatment with pemetrexed has not reported to improve the OS (6) whereas the phase II NVALT 19 trial (7) proved switch maintenance with gemcitabine after first-line platinum-pemetrexed chemotherapy to be efficacious in prolonging progression free survival (PFS) compared with placebo. 
The role of antiangiogenic drugs in MPM along with platinum-based chemotherapy in first-line setting remains controversial. Although the MAPS phase III trial (8) reported an OS improvement with the addition of bevacizumab to platinum-based chemotherapy compared with the chemotherapy alone, the phase III LUME-Meso trial did not reported longer survival with the combination of nintedanib plus pemetrexed-cisplatin compared with chemotherapy plus placebo (9). Notably, neither FDA nor EMA have approved bevacizumab as standard first-line treatment in chemotherapy-naïve patients with unresectable MPM. At the ASCO congress 2020 results of the RAMES phase II trial, comparing the combination of gemcitabineramucirumab versus gemcitabine-placebo as second line treatment, showed 6 months OS improvement for the combination gemcitabine-ramucirumab and a $20 \%$ increase in 12 months OS rate (10).

Clinical trials with molecular targeted agents have not brought much into the treatment strategy since most of them did not demonstrate any efficacy (5), probably because druggable targets are lacking within the peculiar pathogenesis of MPM, mostly characterized by mutations of tumor suppressor genes.

Moreover, there is no standard second-line treatment in MPM after platinum-based chemotherapy. Since immune checkpoint inhibitors (ICI) have revolutionized the treatment strategy and the outcomes of advanced non-small cell lung cancer (NSCLC) (11), several clinical trials were launched also in MPM. The purpose of this review, based on literature data search from 2000 to 2020 , is to summarize the current evidence and rationale for investigating the potential role of immunotherapy in this disease as well as the safety and future therapeutic approaches. Therefore, we asked ourselves what is available in terms of biological data (I), how many trials and their results in terms of efficacy and safety in unresectable (II) and resectable (III) disease, and the currently ongoing trials (IV). We present the following article in accordance with the narrative review reporting checklist (available at http://dx.doi. org/10.21037/tlcr-20-673)

\section{Biological rationale}

The biology of MPM shows significant heterogeneity in both tumor and the microenvironment. A major role of chronic inflammation and local tumor suppression in tumorigenesis has been observed in some experimental models (4) as well as spontaneous regressions attributable to an activation of the immune system (12).
The malignant transformation of mesothelial cells seems to be related to the chronic inflammation of the mesothelium $(13,14)$. According to this hypothesis, inhaled asbestos fibers cannot be broken down by the normal phagocytic process and persist in the pleural cavity, chronically activating macrophages in a process called "frustrated phagocytosis" which leads to the increase of the local immune infiltrate and eventually to the malignant transformation of mesothelial cells (14).

Given the close interaction between the immune infiltrate and mesothelial cells, a number of trials have sought to investigate the role and prognostic value of the immune microenvironment (15-19).

Even though some studies suggested a low mutational load in MPM samples (20) a considerably high number of genetic alterations in MPMs have been detected including point mutations, minute deletions and copy number changes. Such genetic alterations may lead to producing neoantigens, which correlates with the clonal expansion of tumor-infiltrating $\mathrm{T}$ lymphocytes and support the concept of MPM as an immunogenic disease (21-23).

Several studies on T-cell-inhibitory receptors and chemokines have highlighted the prognostic role of lymphocytes and the occurrence of immunosuppression in MPM $(15,16,19)$.

Programmed death (ligand)-1 (PD-L1), the ligand on tumor cells for the PD-1 receptor expressed by activated T and $\mathrm{B}$ cells, has been so far the only predictive biomarker associated to ICI treatment in other thoracic malignancies such as NSCLC. The binding of PD-L1 to PD-1 affects effector T-cell and B-cell function and ultimately leads to exhaustion and apoptosis (24).

In MPM, PD-L1 expression ranges between 40 $45 \%$ (mainly the sarcomatoid MPM subtype) and is heterogeneous, both spatial and temporal, among MPM cells and might vary during treatment $(25,26)$. Different monoclonal antibodies $(\mathrm{mAb})$ can be used for testing PDL1 expression (SP142, SP263, 28-8 and 22C3). In a cohort of $32 \mathrm{MPM}$ it has been reported that the concordance rates between SP142 and 22C3 and between 28-8 and $22 \mathrm{C} 3$ were the highest $(84.4 \%)$, but not for the SP263 $\mathrm{mAb}(27)$.

Similar to other malignancies, PD-L1 expression correlates with poor prognosis, as an example, PD-L1 positive MPM had a shorter median OS compared with PDL1 negative MPM (5 versus 14.5 months, $\mathrm{P}<0.0001)(25,26)$.

Although PD-L1 is a predictive biomarker for ICI efficacy in NSCLC, the predictive role of PD-L1 expression 
in MPM remains controversial and there is no cut-off for defining the tumors with higher probably to obtain a clinical benefit with ICI. However, PD-L1 positive MPM respond better to ICI than negative (28). Recently, the integration between PD-L1 and immune-related genes expression has been suggested as a promising tool to select patients who may benefit from ICIs (29).

Indeed, tumor microenvironment (TME) enriched with cytotoxic $\mathrm{T}$ lymphocytes has been associated with higher levels of macrophages and PD-L1 expression on tumor cells and with aggressive histopathological features, lower response to chemotherapy and shorter OS (30). Finally, in an integrative molecular characterization of MPM was reported a strong expression of the immune-checkpoint gene VISTA in epithelioid MPM, which may have implications for the immune response to MPM and for its immunotherapy strategy (31).

In conclusion, these preclinical data provide a good rationale for developing and testing immunotherapy in MPM.

\section{ICls}

\section{Unresectable disease}

\section{First-line}

In first line setting some data are already available and encouraging for the use of ICIs, while waiting for the larger randomized trials that are still ongoing (Table 1).

In the phase II DREAM study, durvalumab combined with first-line chemotherapy (cisplatin and pemetrexed) was investigated in a single-arm phase II. Overall, 54 patients were enrolled, the primary endpoint PFS at 6 months was $57 \%$, and the objective response rate (ORR) was $48 \%$, with a median duration of response of 6.5 months. Immunerelated adverse events (ir-AEs) of grade 3 and higher, occurred in eight patients (15\%), including lipase elevation $(\mathrm{n}=1)$, pancreatitis $(\mathrm{n}=1)$ and renal impairment $(\mathrm{n}=1)(38)$. Following these results, another phase II single-arm trial evaluating for efficacy in terms of OS durvalumab combined with first-line chemotherapy (39) and a phase III trial, the DREAM3R (40) are currently ongoing.

A recent press-release from the CheckMate743, the open-label, multi-center, randomized phase 3 trial achieved the primary OS endpoint with the combination of nivolumab at $3 \mathrm{mg} / \mathrm{kg}$ every two weeks and ipilimumab at $1 \mathrm{mg} / \mathrm{kg}$ every six weeks compared with standard platinumpemetrexed chemotherapy in patients with previously untreated MPM (41).

Further data are awaited from the BEAT-Meso trial, an open-label, randomized phase III trial comparing the efficacy in terms of PFS and OS as co-primary endpoints of atezolizumab and bevacizumab combined with carboplatin-pemetrexed versus bevacizumab alone combined with carboplatin-pemetrexed (42), and also from the randomized, phase II/III trial evaluating pembrolizumab either alone or combined with firstline chemotherapy (cisplatin and pemetrexed) versus chemotherapy alone in a study by the Canadian Cancer Trials Group (CCTG) (43).

Moreover, dedicated trial in the Japanese population are running: a phase I trial KEYNOTE-A17 assessing the combination of the doublet cisplatin-pemetrexed with pembrolizumab (44) and an exploratory phase II trial on nivolumab with first-line chemotherapy (cisplatin and pemetrexed) (45).

In a summary promising data are raising on the combination of standard chemotherapy and ICI in first line although results should still be awaited.

\section{Second line}

The largest data about the potential role of ICI in MPM comes from second-line setting (see Table 1).

Nivolumab was assessed in two phase II clinical trials, one restricted to the Japanese population and another single-center, Dutch trial; both trials selected the patients according to PD-L1 expression.

The Japanese MERIT study, a single-arm phase II study examined in 34 patients the efficacy and safety of nivolumab monotherapy. The primary endpoint, ORR, was 29.4\% (10 out of 34 enrolled patients); in the sarcomatoid subgroup the ORR reached $67 \%$. Tumor shrinkage was observed, regardless of PD-L1 status (assessed by 28-8 mAb), and the ORR according to PD-L1 expression was $40 \%$ for PD$\mathrm{L} 1 \geq 1 \%$ and $8 \%$ for PD-L $1<1 \%$. Secondary endpoints were median PFS 6.1 months and median OS 17.3 months; concerning safety, twenty-six patients (76\%) experienced treatment-related adverse events. The subgroup analysis of OS and PFS by PD-L1 status exhibited trends, with prolonged OS and PFS for patients with PD-L $1 \geq 1 \%$ s. $<1 \%$ [hazard ratio (HR) for OS 0.542 (95\% CI: $0.208-1.415$, $\mathrm{P}=0.2021)$; HR for PFS 0.725 (95\% CI: 0.316-1.668, $\mathrm{P}=0.4490)](46)$.

Similar results were achieved by the NivoMes trial, single-arm phase II Dutch study enrolling 34 patients that showed $47 \%$ disease control rate (DCR, primary endpoint) 
Table 1 Immune checkpoint inhibitors in MPM

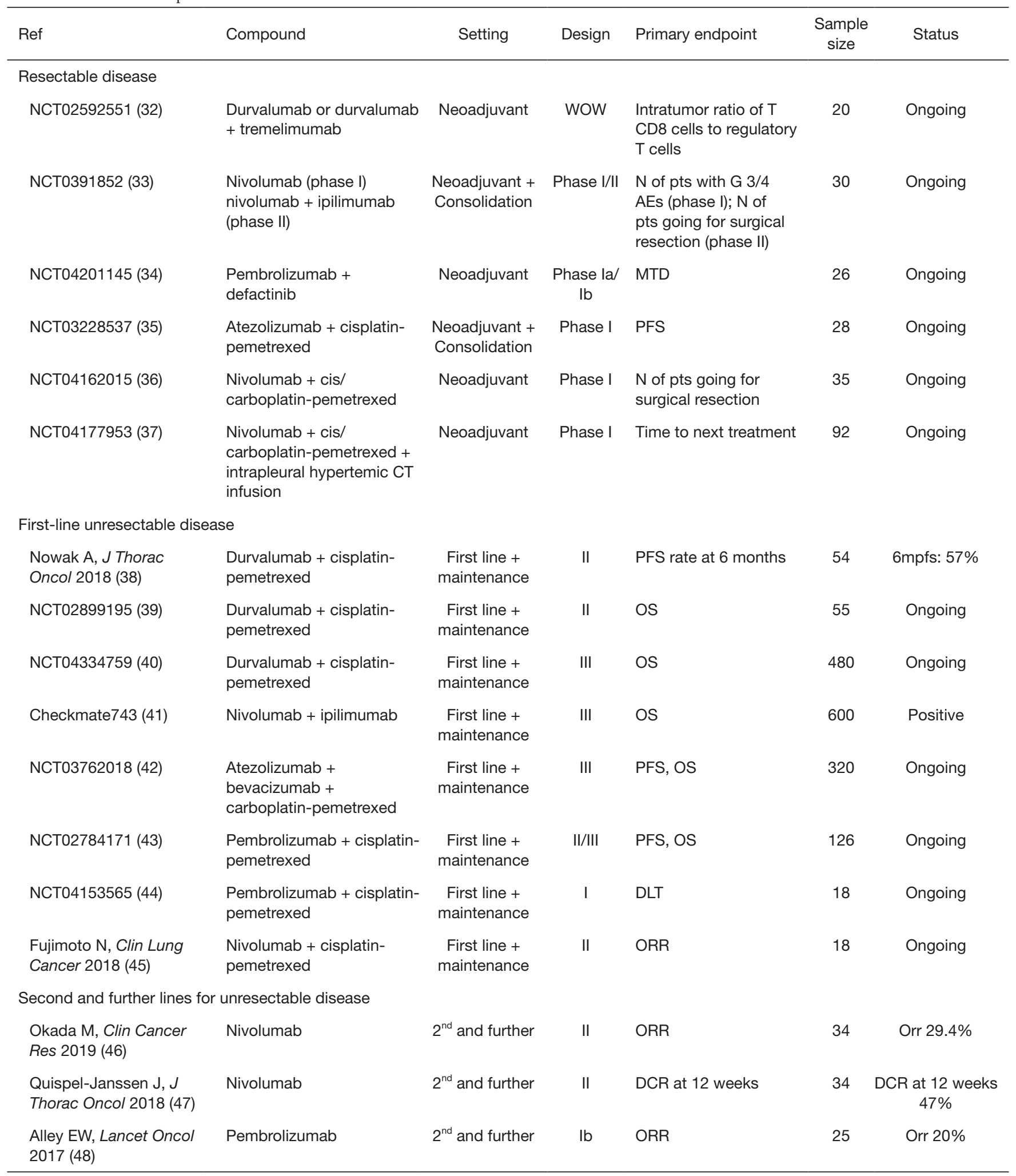

Table 1 (continued) 
Table 1 (continued)

\begin{tabular}{|c|c|c|c|c|c|c|}
\hline Ref & Compound & Setting & Design & Primary endpoint & $\begin{array}{l}\text { Sample } \\
\text { size }\end{array}$ & Status \\
\hline $\begin{array}{l}\text { Desai A, 19th IASLC } \\
\text { World Conference on } \\
\text { Lung Cancer Toronto (49) }\end{array}$ & Pembrolizumab & $2^{\text {nd }}$ and further & II & ORR & 65 & Orr $21 \%$ \\
\hline $\begin{array}{l}\text { Metaxas Y, J Thorac } \\
\text { Oncol } 2018(50)\end{array}$ & Pembrolizumab & $2^{\text {nd }}$ and further & registry & ORR & 93 & Orr $18 \%$ \\
\hline $\begin{array}{l}\text { Hassan R, JAMA } \\
\text { Oncol } 2019 \text { (52) }\end{array}$ & Avelumab & $2^{\text {nd }}$ and further & $\mathrm{lb}$ & ORR & 53 & Orr $9 \%$ \\
\hline $\begin{array}{l}\text { Calabrò L, Lancet Oncol } \\
2013 \text { (53) }\end{array}$ & Tremelimumab & $2^{\text {nd }}$ and further & II & ORR & 29 & Orr $7 \%$ \\
\hline $\begin{array}{l}\text { Calabrò L, Lancet } \\
\text { Respir Med } 2018 \text { (56) }\end{array}$ & $\begin{array}{l}\text { Durvalumab + } \\
\text { tremelimumab }\end{array}$ & $2^{\text {nd }}$ and further & II & ORR & 40 & Orr $28 \%$ \\
\hline $\begin{array}{l}\text { Zalcman G, Lancet } \\
2016(57)\end{array}$ & Nivolumab + ipilimumab & $2^{\text {nd }}$ and further & II & DCR at 12 weeks & 114 & $\begin{array}{c}\text { DCR at } 12 \text { weeks } \\
40 \% \text { nivolumab, } \\
52 \% \text { nivo/ipi }\end{array}$ \\
\hline $\begin{array}{l}\text { Disselhorst MJ, Lancet } \\
\text { Respir Med } 2019 \text { (28) }\end{array}$ & Nivolumab + ipilimumab & $2^{\text {nd }}$ and further & II & ORR & 38 & Orr 29\% \\
\hline $\begin{array}{l}\text { Alley EW, J Clin Oncol } \\
2019 \text { (58) }\end{array}$ & Pembrolizumab + CRS-207 & $2^{\text {nd }}$ and further & II & DCR & 10 & Dcr $11 \%$ \\
\hline NCT03074513 (61) & $\begin{array}{l}\text { Atezolizumab + } \\
\text { bevacizumab }\end{array}$ & $2^{\text {nd }}$ and further & II & ORR & 160 & Ongoing \\
\hline NCT04013334 (62) & Nivolumab + MTG201 & $2^{\text {nd }}$ and further & II & ORR & 12 & Ongoing \\
\hline NCT04166734 (63) & Pembrolizumab + SBRT & $2^{\text {nd }}$ and further & 1 & DLT & 18 & Ongoing \\
\hline NCT04287829 (64) & $\begin{array}{l}\text { Pembrolizumab + } \\
\text { lenvatinib }\end{array}$ & $2^{\text {nd }}$ and further & II & ORR & 36 & Ongoing \\
\hline NCT03760575 (65) & $\begin{array}{l}\text { Pembrolizumab + cisplatin- } \\
\text { pemetrexed + surgery }\end{array}$ & $2^{\text {nd }}$ and further & I & PFS & 20 & Ongoing \\
\hline NCT02959463 (66) & $\begin{array}{l}\text { Radiotherapy -> } \\
\text { pembrolizumab }\end{array}$ & 2nd and further & I & Incidence of AEs & 24 & Ongoing \\
\hline NCT03126630 (67) & $\begin{array}{l}\text { Pembrolizumab +/- } \\
\text { anetumab }\end{array}$ & 2nd and further & $\mathrm{I} / \mathrm{II}$ & $\begin{array}{l}\text { DLT (phase I) ORR } \\
\text { (phase II) }\end{array}$ & 134 & Ongoing \\
\hline NCT02414269 (68) & Pembrolizumab + car-T-cell & 2nd and further & $\mathrm{I} / \mathrm{II}$ & $\begin{array}{l}\text { DLT (phase I) Clinical } \\
\text { Benefit Rate (phase II) }\end{array}$ & 179 & Ongoing \\
\hline
\end{tabular}

MPM, malignant pleural mesothelioma; WOW, window of opportunity; N, number; pts, patients; MTD, maximum tolerated dose; PFS, progression free survival; CT, chemotherapy; OS, overall survival; DLT, dose limiting toxicity; ORR, overall response rate; DCR, disease control rate; SBRT, stereotactic radiotherapy; AEs, adverse events. 
at 12 weeks [ 8 patients with partial response (PR) and 8 with stable disease (SD)], with a median PFS and OS of 2.6 and 11.8 months, respectively (47). The ongoing CONFIRM phase III trial initiated by Cancer Research UK (60) is assessing, in terms of OS, the role of nivolumab compared with placebo in MPM patients with at least progression on 2 previous treatment lines.

Pembrolizumab, another anti-PD1 drug has also been assessed in chemo-refractory MPM patients. The KEYNOTE-028, a phase Ib, single-arm trial only enrolled PD-L 1 positive ( $\geq 2 \%$ by $22 \mathrm{C} 3 \mathrm{mAb}$ ) MPM patients. Pembrolizumab (10 mg/kg Q2W) reported an ORR of $20 \%$, but the DCR was higher compared to nivolumab's one, reaching $72 \%$ ( $52 \%$ had SD), and median PFS and OS of 5.4 months and 18 months, respectively, with 1-year OS of $62.6 \%$, which seemed higher than historical controls. In terms of safety, $16(64 \%)$ patients reported a treatmentrelated adverse event; the most common being fatigue (24\%), nausea (24\%), and arthralgia (20\%). Five (20\%) patients reported grade 3 treatment-related adverse events and $3(12 \%)$ patients required dose interruption because of ir-AEs: 1 (4\%) of 25 each had grade 3 rhabdomyolysis and grade 2 hypothyroidism; grade 3 iridocyclitis, grade 1 erythema multiforme, and grade 3 erythema; and grade 2 infusion-related reaction. No treatment-related deaths or discontinuations occurred (48).

In a second phase II study with pembrolizumab in 65 pre-treated MPM patients not selected by PD-L1 expression, pembrolizumab at $200 \mathrm{mg}$ every three weeks reported an ORR 21\% and median PFS and OS of 4.1 and 11.5 months respectively. High PD-L1 expression was reported in $23 \%$ of tumors but in $45 \%$ of cases PDL1 expression was negative. The ORR was associated with PD-L1 expression: 7\%, 25\%, and $43 \%$ in patients harbouring tumors with PD-L1-expression level of $0 \%, 1-49 \%$ and $\geq 50 \%$ respectively. However, the trial could not determine the optimal threshold for PD-L1 expression (22C3) in correlation with tumor response and no differences in OS were reported according to PD-L1 high versus low/null expression $(\mathrm{P}=0.43)$, whereas higher PD-L1 expression correlated significantly with longer PFS (49).

Finally there is a real-world registry data on the off-label pembrolizumab use in Switzerland and Australia. A total of 93 patients (48 from Switzerland and 45 from Australia) were treated: 68 patients $(73 \%)$ had epithelioid MPM, and $67(72 \%)$ had an ECOG PS of 0 or 1.

PD-L1 expression results were available for 66 patients
(71\%), 68\% were negative ( $<5 \%), 18 \%$ were intermediate (5--49\%), and $14 \%$ were high ( $\geq 50 \%)$ for PD-L1 expression. In the full cohort, the ORR was $18 \%$, the median PFS was 3.1 months, and the median OS was 7.2 months. Compared with intermediate and negative PD-L1 expression, high PDL1 expression was associated with an improved ORR [44\% versus $42 \%$ versus $11 \%(\mathrm{P}=0.01)$ ] and median $\mathrm{PFS}$ [6.2 versus 3.9 versus 2.7 months $(\mathrm{P}=0.04)$ ]. Toxicity was as expected and consistent with other studies (50).

Despite these promising results from phase II, the randomised PROMISE phase III trial did not meet the PFS primary endpoint. The trial enrolled 144 PD-L1 unselected MPM patients with progression to platinumbased chemotherapy and assess the efficacy of secondline pembrolizumab (200 mg Q3W) compared to standard chemotherapy (gemcitabine or vinorelbine). Crossover from chemotherapy arm to ICI arm was allowed at the time of progression. Pembrolizumab did not report longer PFS compared with chemotherapy (2.5 versus 3.4 months, HR $1.06, \mathrm{P}=0.76)$ or longer $\mathrm{OS}(10.7$ versus 11.7 months, HR 1.04, $\mathrm{P}=0.85)$. PD-L1 expression $(<1 \%$ or $\geq 1 \%$ assessed by E1L3N mAb) did not modify influence in the outcome. Treatment-related grade $\geq 3$ adverse events affected $19 \%$ pembrolizumab versus $24 \%$ chemotherapy patients, one fatal per arm. Most common adverse events were fatigue $(19 \%)$ in pembrolizumab whereas nausea $(27 \%)$ and fatigue (31\%) in chemotherapy (51).

Avelumab (10 mg/kg Q2W), an anti-PDL1 drug, was assessed in 53 MPM patients in a phase Ib monotherapy trial (JAVELIN). Avelumab reported an ORR of 9\%, being $19 \%$ in PD-L1-positive ( $\geq 5 \%)$ and $7 \%$ in PD-L1-negative tumors. The median PFS was 4.1 months, whereas the median OS extended to $>10$ months. Five patients (9\%) had grades 3-4 TRAEs, without treatment-related deaths (52).

Focusing on anti-CTLA4, despite preliminary encouraging results with tremelimumab (ORR of 3-7\%) from two single-arm, phase II trials in Europe $(53,54)$, the randomized phase IIb DETERMINE study, revealed that tremelimumab failed to significantly prolong OS compared with that of placebo (7.7 and 7.3 months; HR $0.92,95 \%$ CI: 0.76-1.12) (55), not supporting anti-CTLA4 monotherapy as potential therapeutic strategy in MPM.

Considering the disappointing results of tremelimumab as single-agent, investigators assessed the efficacy in terms of ORR (according to immune-related modified Response Evaluation Criteria in Solid Tumors) of the combination tremelimumab with durvalumab in an open-label, nonrandomised, phase 2 study. In the 40 enrolled patients, the 
ORR was $28 \%$, the immune-related DCR was $65 \%$ and the median response duration was 16.1 months. Median immunerelated PFS was 8.0 months (95\% CI: 6.7-9.3 months), median PFS was 5.7 months (1.7-9.7 months), and median OS was 16.6 months (13.1-20.1 months). Baseline tumour PD-L1 expression did not correlate with the proportion of patients who had an immune-related ORR or immunerelated disease control, with immune-related PFS, or with OS. Treatment-related toxicity was generally manageable and reversible, overall treatment-related AEs rate was $75 \%$ and grade $3-4$ was $18 \%$ (56).

Together with the combination of tremelimumab and durvalumab that appeared active with a good safety profile, also the combination of nivolumab and ipilimumab showed activity.

The French IFCT-1501 MAPS2 was a multicentre, randomised (1:1), non-comparative, open-label, phase 2 trial evaluating, in terms of proportion of patients who achieved 12-week DCR, assessed by masked independent central review, intravenous nivolumab ( $3 \mathrm{mg} / \mathrm{kg}$ bodyweight) every 2 weeks, or intravenous nivolumab $(3 \mathrm{mg} / \mathrm{kg}$ every 2 weeks) plus intravenous ipilimumab ( $1 \mathrm{mg} / \mathrm{kg}$ every 6 weeks), given until progression or unacceptable toxicity. Central randomisation was stratified by histology (epithelioid $v s$. non-epithelioid), treatment line (second line versus third line), and chemo-sensitivity to previous treatment (progression $\geq 3$ months versus $<3$ months after pemetrexed treatment). The 12 -week DCR was $40 \%$ in the nivolumab group and $52 \%$ in the combination group in the intention-to-treat population. Median OS was 11.9 months in the nivolumab arm and 15.9 months in the combination arm, with 2 -year OS of $25.4 \%$ and $31.7 \%$, respectively. Grade 3-4 toxicity rates were $14 \%$ for the nivolumab group and $26 \%$ for the combination group, being fatigue, asymptomatic increase in liver transaminases, and asymptomatic lipase increase. No patients had toxicities leading to death in the nivolumab group, whereas three $5 \%$ of patients in the combination group did (one fulminant hepatitis, one encephalitis, and one acute kidney failure) $(57,69)$. At ESMO congress 2019 , the investigators presented data on treatment hyperprogression (HPD) defined by calculating the tumor growth rate before and during treatment with determining the variation per month ( $\triangle \mathrm{TGR}$ ) or by measuring the tumor growth kinetics (TGK) on treatment and on last treatment before, with TGK ratio (TGKr) calculation. HPD was defined as disease progression (assessed by blinded central reviewer) with $\Delta$ TGR exceeding $50 \%$ or with a TGKr $>2$. With $\Delta$ TGR method, 4 and 2 patients in nivolumab and combination group had HPD respectively, while 7 and 4 patients had HPD by using TGKr. HPD patients with TGKr had a poorer OS than patients with standard progression only in the nivolumab arm [median OS $=1.6(0.8-7.7)$ vs. $4.4(2.4-$ $10.8)$ months, $(\mathrm{P}=0.02$ in Cox model $)$, while no difference was seen with $\triangle$ TGR definition (69).

Similar to this MAPS2 trial, a single-arm study, the INITIATE study evaluated the efficacy of nivolumab and ipilimumab in 35 MPM showing an ORR of $29 \%$, and DCR (primary endpoint) at 12 weeks of $68 \%$ with similar tolerance obtained from the MAPS2 trial. Median PFS was 6.4 months, median OS was not reached and 1-year OS was $64 \%$. Up to $38 \%$ of patients experienced grade $\geq 3$ AEs. A post-hoc analysis for PD-L1 expression (22C3 antibody) was done: 15 (44\%) samples had PD-L1 expression on at least $1 \%$ of tumour cells, of which $12(80 \%)$ were epithelioid, 1 (7\%) was mixed, and 2 (13\%) were sarcomatoid. Responses at 12 weeks for the 15 PD-L1-positive patients (i.e., PD-L1 expression of $\geq 1 \%$ ) were PR in seven (47\%), SD in $6(40 \%)$, and progressive disease in 2 (13\%), which were significantly better than responses for the 19 PD-L1-negative patients, which were PR in $3(16 \%)$, SD in 7 (37\%), and progressive disease in 9 (47\%; $\mathrm{P}=0.018$, linear-by-linear association test). PD-L1 positivity (vs. negativity) was significantly associated with clinical benefit (i.e., $\mathrm{PR}$ or $\mathrm{SD}$ for $>6$ months; $\mathrm{P}=0.037$, Fisher's exact test) (28).

All these data may suggest that the combination of ICI is more suitable than monotherapy in MPM in first line according to the press release from CheckMate 743 trial (41) as well as in second-line setting. Likewise, several combination trials are still ongoing and summarized in Table 1.

Of a note, an interesting combination of pembrolizumab with CRS-207, a live, attenuated, double-deleted Listeria monocytogenes (LADD) engineered to stimulate immune response to mesothelin, showed $11 \%$ DCR and a good safety profile in a phase II, single-arm trial (58).

This study was followed by a multicenter, phase I study investigating anetumab ravtansine, an antibodydrug conjugate of anti-mesothelin antibody linked to maytansinoid DM4, in patients with advanced, metastatic, or recurrent solid tumors, including MPM, known to express the tumor-differentiation antigen mesothelin. There were no drug-related deaths, the most common drug-related AEs being fatigue, nausea, diarrhea, anorexia, vomiting, peripheral sensory neuropathy, and keratitis/ keratopathy. Activity results were interesting (59) and paved the way to an ongoing trial in combination with 
pembrolizumab (67).

\section{Resectable disease}

There are no available data yet on the safety and efficacy of ICIs in resectable MPM since most of trials in early phase (I or II) are still ongoing (see Table 1).

Among them, there are those with combination of ICIs like a window-of-opportunity trial investigating durvalumab either alone or combined with tremelimumab (32) and a phase I/II trial with safety as primary endpoint evaluating for the first 15 patients nivolumab $240 \mathrm{mg}$ administered preoperatively and for 1 year after combined treatment modality (surgery +/- radiotherapy); the design foresees that if no safety concern will be raised the following patients will receive nivolumab $3 \mathrm{mg} / \mathrm{kg}$ combined with ipilimumab $1 \mathrm{mg} / \mathrm{kg}$ (33).

An interesting combination under investigation is the one of pembrolizumab associated with defactinib, a focal adhesion kinase (FAK) inhibitor, as neoadjuvant treatment in a phase I trial (34).

Several other ongoing trials evaluate the combination of anti PD-1 or anti PD-L1 with standard chemotherapy: first, a phase I trial evaluating the safety of the combination of atezolizumab and the doublet cisplatin-pemetrexed followed by surgery with or without radiotherapy and atezolizumab maintenance (35); secondly, a phase I evaluating the number of patients that received surgery after preoperative nivolumab at $360 \mathrm{mg}$ combined with either cisplatin or carboplatin plus pemetrexed (36); and finally a randomized (1:1), phase II trial comparing cis/carboplatin plus pemetrexed after surgery +/- intrapleural hypertemic CT infusion versus the same combination plus nivolumab 480 $\mathrm{mg}$ in terms of time to next treatment (37).

The results of these trials will set the basis to assess the possible further development of these drugs in the early setting, particularly needed since no major advance has been made in the last $10-15$ years.

\section{Vaccines}

Cancer vaccines have not proved effective in the majority of tumors despite their main goal is to induce tumor-specific effector T cells (70).

Only one positive trial with autologous dendritic cell vaccination (DCV), the PMR-MM-002, has been reported in MPM. The study demonstrated the safety and feasibility of tumor lysate-pulsed dendritic cells as therapeutic adjuvants in MPM patients (71).

These results paved the way to several clinical trials with vaccines currently ongoing: a phase II/III randomized clinical trial DENIM (72) with dendritic cell immunotherapy plus best supportive care (BSC) versus BSC alone; a phase I/II on autologous TILs plus IL-2 (73); a phase Ib MESOVAX clinical trial with a combination of autologous DCV and pembrolizumab a (74); a randomized phase II trial with PFS as primary endpoint with nivolumab and ipilimumab with or without UV1 vaccination (so to induce $T$ cell against telomerase)as second line (75).

Few ongoing trials explore the intratumoral injection of compounds: a phase I study of intratumoral PolyICLC, a TLR3 agonist modulating the TME, in patients with potentially resectable MPM (76); a phase I dose escalation clinical trial to investigate if the administration of AdV-tk to patients with MPE followed by valacyclovir and chemotherapy is safe (77); and a phase I clinical trial investigating the side effects and the best dose of local intrapleural measles virus therapy (78).

Several ongoing trials focus on vaccines against the Wilms' Tumor Antigen (WT1), highly overexpressed in MPM as well as several other hematologic and solid tumors thus making it an ideal candidate for a tumor selective cancer vaccine in WT1 expressing malignancies. Although WT1 is a nuclear and cytoplasmic protein that functions as a transcription factor regulating genes involved in cellular proliferation, differentiation, apoptosis, organ development, and sex determination, the protein is processed by the proteasome and the derived peptides are presented on the cell surface making it an attractive target for immunotherapy (79).

Galinpepimut-S is a vaccine targeting WT1 that proved safety and effective when combined with GM-CSF and Montanide in a double-blind, randomized, phase II trial compared to GM-CSF and Montanide alone (80).

Currently a phase I study is investigating the safety of galinpepimut-S combined with nivolumab in patients with WT1-expressing MPM (81).

Finally, the MESODEC trial is currently assessing autologous dendritic cells loaded with WT1 combined with standard chemotherapy (82).

\section{Perspectives}

MPM remains a tumor with a poor prognosis and with limited treatment options. Therefore, results from clinical trials with ICIs were eagerly awaited since they have 
revolutionized the treatment strategy and the outcomes of several tumor types.

So far immunotherapies have demonstrated preclinical and clinical signals of activity and efficacy. Nonetheless, while waiting for the results from the Checkmate 743 trial (41), no clear survival advantage of ICI has been demonstrated by other randomized trials. Among the possible explanations also small sample sizes, methodological constraints like also the exploratory nature of the trials can be counted.

Designing clinical trials with immune therapeutics is definitely challenging considering their mechanism of action that impacts not only the type but also the timing of response (83). For example, modified Response Evaluation Criteria in Solid Tumors for MPM as well as central imaging review were not consistently used to assess response.

Considering the rarity of this tumor, 'small-scale' and 'single-arm' designs, aiming at proving activity in terms of ORR or DCR, are the best ones for drug development but require a valid and solid response assessment. For example, the European Organisation for Research and Treatment of Cancer (EORTC) Lung Cancer Group proved the surrogacy of PFS rate at 9- and 18-week (84). Despite the clinical, methodological and financial advantage, with the exception of expansion cohorts of phase I trials, very limited use of basket and umbrella trials has been made in this disease (85). Also, few trials in MPM have explored the schedule or sequence as well as duration of ICI treatment (86).

Finally, another caution that must be applied is that the majority of patients diagnosed with untreated, unresectable mesothelioma exhibit all expected symptoms at the initial presentation, and thus, do not meet the eligibility criteria to participate in clinical trials. Therefore, study results have to be interpreted cautiously, taking into consideration how each of them can be applied per in-care patient, during daily clinical practices.

Compared with other malignancies, progress in mesothelioma biomarker research is limited, only a few precise biomarkers for ICI efficacy assessments seem to exist in MPM clinical trials, besides PD-L1 expression.

Some of the single-arm ICI studies reveal the correlation between responses and higher PD-L1 expression. However, as insufficient survival data were generated, more established outcome data are needed to confirm the value of PD-L1 immunohistochemistry as a predictive biomarker for the OS effect.
Clearly, single-agent immunotherapy has proven limited benefit, possibly due to MPM complex biology. MPM pathogenesis seems to be essentially driven by inflammation: tumour- associated macrophages are abundantly expressed in MPM stroma and also a low lymphocyte to monocyte ratio in peripheral blood and tissue is reported.

Tumour-associated macrophages can express triggers of checkpoint blockade including PD-L1; at the same time, PD-1 is expressed by natural killer cells (NK cells), immune cells capable of entering coelomic cavities. Therefore, unleashing natural killer cell function from inhibition of tumour-associated macrophages might contribute to the activity of PD-1 targeting monoclonal antibodies in MPM. This strategy of targeting tumour-associated macrophages has the potential to complement PD-1 inhibition (87).

In the close future, the ongoing trials will hopefully confirm the efficacy and safety of immunotherapy combinations both in terms of efficacy and safety and provide new predictive biomarkers.

However, further research needs to be promoted for further and efficient advances in the treatment of this aggressive disease.

\section{Conclusions}

The development of immunotherapeutic agents in MPM is late in time compared to lung cancer and the most frequent tumors.

Nevertheless, there is a promising biological rationale for evaluating not only ICIs, both alone and combined with chemotherapy and other biological agents, but also cancer vaccines.

Encouraging data have emerged from the early clinical trials completed so far and more is awaited since the positive results from the Checkmate743 (35).

Since no major treatment advance has been made in the last ten years, the scientific community and mostly patients have high expectations on the efficacy results of these compounds in a disease with such a bad prognosis whose incidence is still raising.

\section{Acknowledgments}

Funding: None.

\section{Footnote}

Provenance and Peer Review: This article was commissioned 
by the editorial office, Translational Lung Cancer Research for the series "Immunotherapy in other thoracic malignancies and uncommon populations". The article has undergone external peer review.

Reporting Checklist: The authors have completed the Narrative Review reporting checklist. Available at http:// dx.doi.org/10.21037/tlcr-20-673

Conflicts of Interest: All authors have completed the ICMJE uniform disclosure form (available at http://dx.doi. org/10.21037/tlcr-20-673). The series "Immunotherapy in other thoracic malignancies and uncommon populations" was commissioned by the editorial office without any funding or sponsorship. JR served as the unpaid Guest Editor of the series and serves as an unpaid editorial board member of Translational Lung Cancer Research from Sep 2019 to Sep 2021. JR reports payment or honoraria from PFIZER, ASTRA-ZENECA, BMS, MSD, OSE-IMMUNOTHERAPEUTICS, travel support from ASTRA-ZENECA, participation in GENMAB, MSD, BMS and EORTC LUNG CANCER GROUP SECRETARY. The authors have no other conflicts of interest to declare.

Ethical Statement: The authors are accountable for all aspects of the work in ensuring that questions related to the accuracy or integrity of any part of the work are appropriately investigated and resolved.

Open Access Statement: This is an Open Access article distributed in accordance with the Creative Commons Attribution-NonCommercial-NoDerivs 4.0 International License (CC BY-NC-ND 4.0), which permits the noncommercial replication and distribution of the article with the strict proviso that no changes or edits are made and the original work is properly cited (including links to both the formal publication through the relevant DOI and the license). See: https://creativecommons.org/licenses/by-ncnd/4.0/.

\section{References}

1. Gatta G, Capocaccia R, Botta L, et al. Burden and centralised treatment in Europe of rare tumours: results of RARECAREnet-a population-based study. Lancet Oncol 2017;18:1022-39.

2. Siesling S, van der Zwan JM, Izarzugaza I, et al, Rare thoracic cancers, including peritoneum mesothelioma. Eur J Cancer 2012;48:949-60.

3. Vogelzang NJ, Rusthoven JJ, Symanowski J, et al. Phase III study of pemetrexed in combination with cisplatin versus cisplatin alone in patients with malignant pleural mesothelioma. J Clin Oncol 2003;21:2636-44.

4. Yap TA, Aerts JG, Popat S, Fennell DA. Novel insights into mesothelioma biology and implications for therapy. Nat Rev Cancer 2017;17:475-88.

5. Nicolini F, Bocchini M, Bronte G, et al. Malignant Pleural Mesothelioma: State-of-the-Art on Current Therapies and Promises for the Future. Front Oncol 2020;9:1519.

6. Dudek AZ, Wang XF, Gu L, et al. Randomized phase 2 study of maintenance pemetrexed (Pem) versus observation (Obs) for patients (pts) with malignant pleural mesothelioma (MPM) without progression after first-line chemotherapy: Cancer and Leukemia Group B (CALGB) 30901 (Alliance). J Clin Oncol 2019;37:abstr 8517.

7. Burgers SA, de Gooijer C, Cornelissen R, et al. Switch maintenance gemcitabine after first-line chemotherapy in patients with malignant mesothelioma: A multicenter open label phase II trial (NVALT19), Ann Oncol 2019;30:931-2

8. Scherpereel A, Mazieres J, Greillier L, et al. Second- or third-line nivolumab (Nivo) versus nivo plus ipilimumab (Ipi) in malignant pleural mesothelioma (MPM) patients: Results of the IFCT-1501 MAPS2 randomized phase II trial. J Clin Oncol 2017;35:abstr LBA8507.

9. Scagliotti GV, Gaafar R, Nowak AK, et al. Nintedanib in combination with pemetrexed and cisplatin for chemotherapy-naive patients with advanced malignant pleural mesothelioma (LUME-Meso): a double-blind, randomised, placebo-controlled phase 3 trial. Lancet Respir Med 2019;7:569-80.

10. Pagano M, Ceresoli GL, Zuccali PA, et al. Randomized phase II study on gemcitabine with or without ramucirumab as second-line treatment for advanced malignant pleural mesothelioma (MPM): Results of Italian Rames Study. J Clin Oncol 2020;38:abstr 9004.

11. Planchard D, Popat S, Kerr K, et al. Metastatic non-small cell lung cancer: ESMO Clinical Practice Guidelines for diagnosis, treatment and follow-up. Ann Oncol 2018;29:iv192-iv237.

12. Robinson BW, Robinson C, Lake RA. Localised spontaneous regression in mesothelioma-possible immunological mechanism. Lung Cancer 2001;32:197-201.

13. Carbone M, Yang H. Molecular pathways: targeting mechanisms of asbestos and erionite carcinogenesis in mesothelioma. Clin Cancer Res 2012;18:598-604. 
14. Bograd AJ, Suzuki K, Vertes E, et al. Immune responses and immunotherapeutic interventions in malignant pleural mesothelioma. Cancer Immunol Immunother 2011;60:1509-27.

15. Anraku M, Cunningham KS, Yun Z, et al. Impact of tumor-infiltrating $\mathrm{T}$ cells on survival in patients with malignant pleural mesothelioma. J Thorac Cardiovasc Surg 2008;135:823-9.

16. Yamada N, Oizumi S, Kikuchi E, et al. CD8+ tumorinfiltrating lymphocytes predict favorable prognosis in malignant pleural mesothelioma after resection. Cancer Immunol Immunother 2010;59:1543-9.

17. Burt BM, Rodig SJ, Tilleman TR, et al. Circulating and tumor-infiltrating myeloid cells predict survival in human pleural mesothelioma. Cancer 2011;117:5234-44.

18. Suzuki K, Kadota K, Sima CS, et al. Chronic inflammation in tumor stroma is an independent predictor of prolonged survival in epithelioid malignant pleural mesothelioma patients. Cancer Immunol Immunother 2011;60:1721-8.

19. Ujiie H, Kadota K, Nitadori JI, et al. The tumoral and stromal immune microenvironment in malignant pleural mesothelioma: A comprehensive analysis reveals prognostic immune markers. Oncoimmunology 2015;4:e1009285.

20. Bueno R, Stawiski EW, Goldstein LD, et al. Comprehensive genomic analysis of malignant pleural mesothelioma identifies recurrent mutations, gene fusions and splicing alterations Nat Genet 2016;48:407-16.

21. Mansfield AS, Peikert T, Smadbeck JB, et al. Neoantigenic potential of complex chromosomal rearrangements in mesothelioma. J Thorac Oncol 2019;14:276-87.

22. Yoshikawa Y, Emi M, Hashimoto-Tamaoki T, et al. High-Density array- CGH with targeted NGS unmask multiple noncontiguous minute deletions on chromosome 3p21 in mesothelioma. Proc Natl Acad Sci U S A 2016;113:13432-7.

23. Carbone M, Yang H, Gaudino G. Does chromothripsis make mesothelioma an immunogenic cancer? J Thorac Oncol 2019;14:157-9.

24. Gibbons Johnson RM, Dong H. Functional expression of programmed death-ligand 1 (B7-H1) by immune cells and tumour cells. Front Immunol 2017;8:961.

25. Mansfield AS, Roden AC, Peikert T, et al. B7-H1 expression in malignant pleural mesothelioma is associated with sarcomatoid histology and poor prognosis. J Thorac Oncol 2014;9:1036-40.

26. Terra SBSP, Mansfield AS, Dong H, et al. Temporal and spatial heterogeneity of programmed cell death 1-ligand 1 expression in malignant mesothelioma. Oncoimmunology 2017;6:e1356146.

27. Watanabe T, Okuda K, Murase T, et al. Four immunohistochemical assays to measure the PD-L1 expression in malignant pleural mesothelioma. Oncotarget 2018;9:20769-80.

28. Disselhorst MJ, Quispel-Janssen J, Lalezari F, et al. Ipilimumab and nivolumab in the treatment of recurrent malignant pleural mesothelioma (initiate): results of a prospective, single-arm, phase 2 trial. Lancet Respir Med 2019;7:260-70.

29. Patil NS, Righi L, Koeppen H, et al. Molecular and Histopathological Characterization of the Tumor Immune Microenvironment in Advanced Stage of Malignant Pleural Mesothelioma. J Thorac Oncol 2018;13:124-33.

30. Pasello G, Zago G, Lunardi F, et al. Malignant pleural mesothelioma immune microenvironment and checkpoint expression: correlation with clinical-pathological features and intratumor heterogeneity over time. Ann Oncol 2018;29:1258-65.

31. Hmeljak J, Sanchez-Vega F, Hoadley KA, et al. Integrative Molecular Characterization of Malignant Pleural Mesothelioma. Cancer Discov 2018;8:1548-65.

32. NCT02592551. Available online: www.clinicaltrials.gov 33. NCT0391852. Available online: www.clinicaltrials.gov

34. NCT04201145. Available online: www.clinicaltrials.gov

35. NCT03228537. Available online: www.clinicaltrials.gov

36. NCT04162015. Available online: www.clinicaltrials.gov

37. NCT04177953. Available online: www.clinicaltrials.gov

38. Nowak A, Kok P, Lesterhuis W, et al. OA08.02 DREAM - A Phase 2 Trial of Durvalumab with First Line Chemotherapy in Mesothelioma: Final Result. J Thorac Oncol 2018;13:S338-9.

39. NCT02899195. Available online: www.clinicaltrials.gov

40. NCT04334759. Available online: www.clinicaltrials.gov

41. Available online: https://news.bms.com/press-release/ corporatefinancial-news/bristol-myers-squibb-announcespositive-topline-result-pivotal

42. NCT03762018. Available online: www.clinicaltrials.gov 43. NCT02784171. Available online: www.clinicaltrials.gov 44. NCT04153565. Available online: www.clinicaltrials.gov

45. Fujimoto N, Aoe K, Kozuki T, et al. A phase II trial of first-line combination chemotherapy with cisplatin, pemetrexed, and nivolumab for unresectable malignant pleural mesothelioma: a study protocol. Clin Lung Cancer 2018;19:e705-7.

46. Okada M, Kijima T, Aoe K, et al. Clinical Efficacy and Safety of Nivolumab: Results of a Multicenter, Openlabel, Single-arm, Japanese Phase II study in Malignant 
Pleural Mesothelioma (MERIT). Clin Cancer Res 2019;25:5485-92.

47. Quispel-Janssen J, van der Noort V, de Vries JF, et al. Programmed death 1 blockade with nivolumab in patients with recurrent malignant pleural mesothelioma. J Thorac Oncol 2018;13:1569-76.

48. Alley EW, Lopez J, Santoro A, et al. Clinical safety and activity of pembrolizumab in patients with malignant pleural mesothelioma (KEYNOTE-028): preliminary results from a non-randomised, open- label, phase 1B trial. Lancet Oncol 2017;18:623-30.

49. Desai A, Karrison T, Rose B, et al. Phase II trial of pembrolizumab (NCT02399371) in previously treated malignant mesothelioma: final analysis, in IASLC (ED). 19th IASLC World Conference on Lung Cancer Toronto, Canada, 2018.

50. Metaxas Y, Rivalland G, Mauti LA, et al. Pembrolizumab as Palliative Immunotherapy in Malignant Pleural Mesothelioma. J Thorac Oncol 2018;13:1784-91.

51. Popat S, Curioni A, Polydoropoulou V, et al. A multicentre randomized phase III trial comparing pembrolizumab (p) vs single agent chemotherapy (ct) for advanced pre-treated malignant pleural mesothelioma (mpm) - results from the European Thoracic Oncology Platform (ETOP 9-15) PROMISE-MESO trial. Ann Oncol 2019;30:v851-v934.

52. Hassan R, Thomas A, Nemunaitis JJ, et al. Efficacy and safety of avelumab treatment in patients with advanced unresectable mesothelioma: phase $1 \mathrm{~B}$ results from the javelin solid tumor trial. JAMA Oncol 2019;5:351-7.

53. Calabrò L, Morra A, Fonsatti E, et al. Tremelimumab for patients with chemotherapy-resistant advanced malignant mesothelioma: an open-label, single-arm, phase 2 trial. Lancet Oncol 2013;14:1104-11.

54. Calabrò L, Morra A, Fonsatti E, et al. Efficacy and safety of an intensified schedule of tremelimumab for chemotherapy-resistant malignant mesothelioma: an open-label, single-arm, phase 2 study. Lancet Respir Med 2015;3:301-9.

55. Maio M, Scherpereel A, Calabrò L, et al. Tremelimumab as second- line or third-line treatment in relapsed malignant mesothelioma (DETERMINE): a multicentre, international, randomised, double-blind, placebocontrolled phase 2B trial. Lancet Oncol 2017;18:1261-73.

56. Calabrò L, Morra A, Giannarelli D, et al. Tremelimumab combined with durvalumab in patients with mesothelioma (NIBIT-MESO-1): an open-label, non-randomised, phase 2 study. Lancet Respir Med 2018;6:451-60.

57. Zalcman G, Mazieres J, Margery J, et al. Bevacizumab for newly diagnosed pleural mesothelioma in the Mesothelioma Avastin Cisplatin Pemetrexed Study (MAPS): a randomised, controlled, open-label, phase 3 trial. Lancet 2016;387:1405-14.

58. Alley EW, Tanvetyanon T, Jahan TM, et al. A phase II single-arm study of CRS-207 with pembrolizumab (pembro) in previously treated malignant pleural mesothelioma (MPM). J Clin Oncol 2019;37:29.

59. Hassan R, Blumenschein GR Jr, Moore KN, et al. First-in-Human, Multicenter, Phase I Dose-Escalation and Expansion Study of Anti-Mesothelin AntibodyDrug Conjugate Anetumab Ravtansine in Advanced or Metastatic Solid Tumors. J Clin Oncol 2020;38:1824-35.

60. NCT03063450. Available online: www.clinicaltrials.gov 61. NCT03074513. Available online: www.clinicaltrials.gov 62. NCT04013334. Available online: www.clinicaltrials.gov 63. NCT04166734. Available online: www.clinicaltrials.gov 64. NCT04287829. Available online: www.clinicaltrials.gov 65. NCT03760575. Available online: www.clinicaltrials.gov 66. NCT02959463. Available online: www.clinicaltrials.gov 67. NCT03126630. Available online: www.clinicaltrials.gov 68. NCT02414269. Available online: www.clinicaltrials.gov

69. Zalcman G, Mazieres J, Greiller L, et al. Second/third-line nivolumab vs nivo plus ipilimumab in malignant pleural mesothelioma: Long-term results of IFCT-1501 MAPS2 phase IIR trial with a focus on hyperprogression (HPD). Ann Oncol 2019;30:v747-55.

70. Palucka K, Banchereau J. Cancer immunotherapy via dendritic cells. Nat Rev Cancer. 2012;12:265-77.

71. Cornelissen R, Hegmans JP, Maat AP, et al. Extended tumour control after dendritic cell vaccination with lowdose cyclophosphamide as adjuvant treatment in patients with malignant pleural mesothelioma. Am J Respir Crit Care Med 2016;193:1023-31.

72. NCT03610360. Available online: www.clinicaltrials.gov

73. Doherty M, Leighl NB, Feld R, et al. Phase I/II study of tumour-infiltrating lymphocyte (TIL) infusion and low-dose interleukin-2 (IL-2) in patients with advanced malignant pleural mesothelioma (MPM). J Clin Oncol 2015;33:abstr TPS7586.

74. NCT03546426. Available online: www.clinicaltrials.gov

75. NCT04300244. Available online: www.clinicaltrials.gov

76. NCT04345705. Available online: www.clinicaltrials.gov

77. NCT01997190. Available online: www.clinicaltrials.gov

78. NCT01503177. Available online: www.clinicaltrials.gov

79. Amin KM, Litzky LA, Smythe WR, et al. Wilms' tumor 1 susceptibility (WT1) gene products are selectively expressed in malignant mesothelioma. Am J Pathol 
1995;146:344-56.

80. Zauderer MG, Tsao AS, Dao T, et al. A Randomized Phase II Trial of Adjuvant Galinpepimut-S, WT-1 Analog Peptide Vaccine, after Multimodality Therapy for Patients with Malignant Pleural Mesothelioma. Clin Cancer Res 2017;23:7483-9.

81. NCT04040231. Available online: www.clinicaltrials.gov

82. NCT02649829. Available online: www.clinicaltrials.gov

83. Menis J, Litière S, Tryfonidis K, et al. The European Organization for Research and Treatment of Cancer perspective on designing clinical trials with immune therapeutics. Ann Transl Med 2016;4:267.

84. Hasan B, Greillier L, Pallis A, et al. Progression free survival rate at 9 and 18 weeks predict overall survival in patients with malignant pleural mesothelioma: an

Cite this article as: Menis J, Pasello G, Remon J. Immunotherapy in malignant pleural mesothelioma: a review of literature data. Transl Lung Cancer Res 2021;10(6):2988-3000. doi: $10.21037 /$ tlcr-20-673 individual patient pooled analysis of 10 European Organisation for Research and Treatment of Cancer Lung Cancer Group studies and an independent study validation. Eur J Cancer 2014;50:2771-82.

85. Menis J, Hasan B, Besse B. New clinical research strategies in thoracic oncology: clinical trial design, adaptive, basket and umbrella trials, new end-points and new evaluations of response. Eur Respir Rev 2014;23:367-78.

86. Remon J, Menis J, Aspeslagh S, et al. Treatment duration of checkpoint inhibitors for NSCLC. Lancet Respir Med 2019;7:835-7.

87. Ceresoli GL, Mantovani A. Immune checkpoint inhibitors in malignant pleural mesothelioma. Lancet Oncol 2017;18:559-61. 